\title{
MÉXICO, EL DERRUMBE DE UN RÉGIMEN Y LA POLÍTICA EXTERIOR
}

\author{
MEXICO, THE COLLAPSE OF A REGIME \\ AND FOREIGN POLICY
}

\section{LE MEXIQUE: L'EFFONDREMENT D'UN RÉGIME, LA POLITIQUE ÉTRANGÈRE}

\author{
Olga Pellicer Silva* \\ Instituto Tecnológico Autónomo de México, ITAM \\ opellicer@itam.mx
}

Resumen: En el presente ensayo se analizan los rasgos sobresalientes del periodo 2012-2018, en que las instituciones políticas entraron en crisis y el país enfrentó retos inéditos en la relación con Estados Unidos. Examina las condiciones en que se llevó a cabo la política exterior de México, tanto internas como externas, y su incidencia en las decisiones políticas. Como resultado de ello se divide el sexenio en tres partes derivadas de los hechos sustantivos que lo marcaron, para referirse en la parte final a la actividad del presidente electo en los meses previos a la toma de posesión.

Palabras clave: Enrique Peña Nieto; reformas estructurales; Ayotzinapa;

Donald Trump; Luis Videgaray, relación con Estados Unidos; Andrés Manuel López Obrador; México; política exterior.

AвSTRACT: This article analyzes the salient features of the 2012-2018 administration, during which political institutions entered crisis and the country faced unprecedented challenges in its relationship with the United States. It examines the conditions under which Mexico's foreign policy, both internal and external, was implemented, and its impact on political decisions. As a result, it divides the administration into three parts on the basis of the major events that defined it, before describing in the final section the activity of the president-elect in the months prior to taking office.

* Este trabajo está basado en diversas investigaciones publicadas por la autora en capítulos de libros y artículos citados en la bibliografía. 
Keywords: Enrique Peña Nieto; structural reforms; Ayotzinapa;

Donald Trump; Luis Videgaray; relationship with the United States;

Andrés Manuel López Obrador; Mexico; foreign policy.

\section{Traducción de Gonzalo Celorio Morayta}

RÉsumé: Ce texte analyse les traits saillants de la période 2012-2018, durant laquelle les institutions politiques du Mexique sont entrées en crise, juste au moment où le pays devait faire face à des défis jamais vus dans ses rapports avec les États-Unis. On décrit les conditions, internes et internationales, dans lesquelles s'est déroulée la politique étrangère mexicaine, ainsi que leur influence sur les décisions qui ont été prises. Dans ce but, on distingue trois étapes le long du gouvernement en question, suivant les faits essentiels qui ont marqué chacune d'elles. La dernière partie de l'article fait allusion aussi aux activités du successeur de Peña Nieto -le président élu, López Obrador- durant les mois qui ont précédé son accès au pouvoir.

Mots clefs: Enrique Peña Nieto; réformes structurelles; Ayotzinapa;

Donald Trump; Luis Videgaray; rapports Mexique-États-Unis; Andrés Manuel López Obrador; Mexique; politique étrangère.

Traducción de BERNARDo MABIRE

Fecha de recepción: diciembre de 2018

Fecha de aceptación: febrero de 2019 
E L sexenio 2012-2018 pasará a la historia como un periodo excepcional de transformaciones en la vida interna y externa de México. Por ello, el contenido de este artículo tiene que ver más con las condiciones en que se llevó a cabo la política exterior que con las manifestaciones específicas de la misma. Lo primero es, en mi opinión, más significativo que lo segundo. En particular porque ayuda a entender los objetivos que se persiguieron en las relaciones con el exterior en momentos de cambios sustantivos en el interior y el exterior del país, y las posibilidades que se tuvieron, o no, de alcanzarlos.

El gobierno de Enrique Peña Nieto (EPN, 2012-2018) inició su mandato en un ambiente de relativa tranquilidad. La disputa electoral había sido menos ríspida que en el cambio de gobierno de 2006; el ánimo de gran parte de la población era amable, apoyado por la imagen de la esposa del presidente, una actriz conocida gracias al éxito de una telenovela que había conmovido a millones de mexicanos. Nadie sospechaba que seis años después, el partido que llevó a EPN al poder, factótum de la política mexicana durante cerca de ochenta años, estaría prácticamente disuelto, que se habrían desmoronado las fuerzas principales representadas en aquel entonces en el Congreso y que un movimiento social nuevo, conocido como Morena, llevaría al poder, en medio de un entusiasmo delirante, a un líder social que muchos consideraban había terminado su carrera política en 2012.

En el ámbito internacional, los cambios fueron igualmente sorprendentes. La llegada a la presidencia de Estados Unidos de Donald Trump, una figura inesperada, proveniente de la especulación financiera y el mundo del entretenimiento, modificó en poco tiempo el papel de este país como líder del orden mundial liberal imperante en el mundo occidental. Un mundo en caos era la apreciación formulada de diversas maneras en la mayoría de los análisis sobre la situación internacional. Sin duda, el mundo en la era de Trump trastoca todo un entramado de alianzas establecidas, formas diplomáticas, valores y fines compartidos, que pare- 
cía sólido hasta antes de su llegada a la presidencia estadounidense. En lo que toca a México y su relación con los otros países norteamericanos, la llegada de Trump dejó muy atrás la idea de una América del Norte como región con un destino común.

Dentro de esos cambios inesperados que caracterizan el sexenio bajo estudio, la política exterior mexicana no siguió una evolución lineal. Se divide en tres periodos claramente diferenciados. El primero corresponde al momento de la llegada al poder de EPN, en diciembre de 2012, y llegaría hasta septiembre de 2014. Ese primer año y medio constituiría una época de logros en que se alcanzaron metas significativas como las reformas estructurales que, según las expectativas del grupo en el poder, abrían el camino a la modernización y al crecimiento económico del país.

El segundo periodo abarcaría de finales de 2014 a mediados de 2015, cuando la irrupción de problemas políticos internos y cambios en la economía internacional impactaron duramente en el ánimo triunfalista con que inició el sexenio. La imagen de un México violento, esta vez más vinculado con la corrupción de los dirigentes políticos, volvió a dominar las noticas sobre el país en los medios de comunicación internacionales. El encanto de los dos primeros años se desvaneció y no pudo recuperarse en todo el resto del sexenio.

El tercer y último periodo pareció estar dominado por el reto inesperado que significó para la presidencia mexicana la campaña y el triunfo electoral de Donald Trump. Su antimexicanismo, sus ataques a los migrantes y su propósito de levantar un gran muro que separara a los dos países dio el tono a uno de las épocas más complejas y difíciles de la diplomacia mexicana. La búsqueda del entendimiento necesario con Estados Unidos tuvo como principal protagonista a Luis Videgaray, uno de los hombres fuertes de la época de Enrique Peña Nieto, que dio un toque personalista a la relación con Estados Unidos no visto con anterioridad. Como veremos más adelante, los resultados obtenidos fueron ambivalentes. 
Los últimos cinco meses del gobierno de EPN transcurrieron bajo la sombra del arrollador triunfo electoral del Partido Movimiento de Regeneración Nacional (Morena) el $1^{\circ}$ de julio de 2018, el cual llevó al poder a Andrés Manuel López Obrador (AMLO), abriendo con ello una etapa novedosa de enormes consecuencias en la política nacional e internacional de México. Aunque formalmente el presidente electo tomó posesión el $1^{\circ}$ de diciembre, el hecho fue que, bajo la fuerza de circunstancias en ocasiones fuera de su control como candidato vencedor, AMLO comenzó a ejercer como presidente en funciones prácticamente días después del $1^{\circ}$ de julio, compartiendo y en ocasiones dejando en la sombra al gobierno de Peña Nieto. El relato de esa etapa novedosa e inédita en la historia de la política exterior mexicana es el objetivo de la última parte de este ensayo.

EL "MOMENTO MEXICANO", DICIEMBRE DE 2012-SEPTIEMBRE DE 2014

Cuando Enrique Peña Nieto llegó a la presidencia, en diciembre de 2012, los retos que tenía por delante estaban bien identificados. Desde el punto de vista económico, se trataba de revertir el lento crecimiento de la economía que, con altibajos, venía situándose por debajo de los requerimientos del país en materia de creación de empleo, construcción de infraestructura y satisfacción de las necesidades básicas de un amplio sector de la población. De acuerdo con estimaciones del Consejo Nacional de Evaluación Social (Coneval), alrededor de $45 \%$ de la población se encontraba por debajo de los umbrales de pobreza. En 2012, el número de personas en situación de pobreza extrema alcanzó la cifra de 11.5 millones, equivalentes a $9.8 \%$ de la población. ${ }^{1}$

${ }^{1}$ Para una visión general de los factores internos y externos que condicionaron la política exterior durante el sexenio, véase Guadalupe González G. y Olga Pellicer "El posicionamiento internacional de México en 
Desde el punto de vista político, el país atravesaba una de las peores crisis de seguridad y violencia de los últimos cincuenta años. La lucha contra el crimen organizado emprendida desde 2007 por el presidente Felipe Calderón Hinojosa (2006-2012) desencadenó un enfrentamiento entre carteles de la droga que produjo alrededor de 122000 muertos en el periodo en que fungió como presidente. Las labores de las instituciones encargadas de combatir a los narcotraficantes encontraban serios obstáculos por el riesgo de ser infiltradas gracias a los grandes recursos financieros de las organizaciones criminales asociadas al muy rentable negocio del narcotráfico. Los medios de comunicación internacionales dieron seguimiento detallado a la situación anterior. La imagen de México en el mundo se deterioró y llegó a uno de sus niveles más bajos.

El gobierno entrante de EPN enfrentó esa problemática de diversas maneras. Por una parte, privilegió un cambio de imagen en el ámbito internacional mediante la promoción de "el momento mexicano", un eslogan atinado en el contexto de los graves problemas que atravesaban economías emergentes como la de Brasil y, en contrapartida, el buen crecimiento de México en 2012 y la estabilidad de sus principales variables económicas (tipo de cambio, control de la inflación, altas reservas internacionales y balanza de pagos). También se modificó la narrativa gubernamental sobre el crimen y la violencia, evitando que las informaciones al respecto se convirtieran en noticias de primera plana en los medios de comunicación internacionales.

Los mejores argumentos para proyectar una imagen nueva, capaz de levantar expectativas sobre las oportunidades ofrecidas por México, fueron sin duda las habilidades del nuevo gobierno para articular un amplio acuerdo entre las principales fuerzas políticas representadas en el Congreso y

el segundo decenio del siglo xxI", en Ricardo Lagos y Enrique Iglesias (coords.), América Latina, China y Estados Unidos. Perspectivas latinoamericanas, Chile, Fondo de Cultura Económica, 2015, pp. 213-272. 
el anuncio de la puesta en marcha de diversas reformas estructurales que abrirían la puerta a una verdadera transformación para el crecimiento y modernización del país.

Llevar a cabo reformas que destrabasen la inercia de problemas que venían entorpeciendo el funcionamiento de la economía mexicana era una preocupación compartida por las elites políticas y académicas del país desde hacía varios años. El terreno era fértil para proponer un gran "Pacto por México" de naturaleza muy incluyente, en el que se recogiese una amplia gama de preocupaciones compartidas por los tres grandes partidos con representación en el Congreso: el Partido Revolucionario Institucional (PRI), el Partido Acción Nacional (PAN) y el Partido de la Revolución Democrática (PRD).

Está fuera de los límites de este ensayo analizar los efectos de la búsqueda de consensos entre esos partidos respecto al contenido final de las reformas, un tanto diluido en algunas de éstas, marcadamente en la reforma fiscal, así como en el comportamiento y credibilidad de los dirigentes que comenzaron a tomar compromisos al interior del Pacto. Algunos de los problemas que surgieron más tarde (como la creación de Morena) tuvieron su origen en las distancias que, como resultado de tales compromisos, se crearon entre las bases y los dirigentes de los partidos políticos. La distancia y falta de confianza en los liderazgos se originó desde aquellos momentos, así como las dificultades para encauzar demandas sociales que quedaron pendientes. También queda fuera de los límites del ensayo reflexionar sobre las desventajas de haber llevado a cabo un número tan grande de reformas, aprobadas con sorprendente rapidez. Si ello permitió aprovechar el "momento mexicano" a cambio dejó una serie de incertidumbres sobre cómo se iba a transitar de la letra de las reformas, los reglamentos y las nuevas instituciones a su implementación y puesta en marcha.

Las reformas fueron heterogéneas por el nivel de impacto económico que tuvieron y el grado de conflicto y choque de intereses que provocaron. Dos de ellas merecen destacarse: la relativa a las telecomunicaciones (que buscó romper el 
monopolio ejercido por dos grandes gigantes como Televisa y Telmex), y la reforma energética, quizá la de mayor impacto en la vida nacional. El meollo de esta última fue la apertura de la actividad en materia de hidrocarburos a la iniciativa privada nacional y extranjera, lo que representó un giro de 180 grados en el pensamiento que por más de setenta años dominó el imaginario político de la mayoría de los mexicanos. Tomando en cuenta la riqueza petrolera existente en las aguas profundas y ultraprofundas del Golfo de México, así como la importancia de los yacimientos de gas de lutitas en el norte del país, la medida tuvo como consecuencia un alto costo político para quienes la promovieron y una posibilidad de ofrecer a los inversionistas atractivos para invertir en México.

Cabría detenerse aquí y preguntarse sobre el papel específico que ocupó la política exterior en el vendaval de actividades desatado por la elaboración y aprobación de las reformas estructurales. Lo cierto es que casi no estuvo presente. A pesar de que los factores externos serían fundamentales para los objetivos que buscaban, como acelerar mediante inversión extranjera la exploración y la exportación de hidrocarburos, la política exterior no tuvo un apartado dentro del gran número de actividades establecidas en el Pacto por México. La única acción prevista en materia internacional fue la creación de los Institutos México, que entrarían en funcionamiento en el segundo semestre de 2013. Estos Institutos nunca se materializaron; la única reflexión seria al respecto se refirió al nombre que debían llevar. ${ }^{2}$

Tomando en cuenta esa omisión, se esperaba con interés algún discurso o toma de posición del nuevo canciller, José Antonio Meade, sobre las continuidades o cambios que se darían respecto de la política exterior seguida en los doce años previos por parte de gobiernos emanados del PAN. Di-

${ }^{2}$ Para la política exterior en el primer periodo del sexenio véase "La política exterior de México bajo un nuevo presidente", Centro de Estudios Internacionales de Barcelona, сіDов, Anuario Internacional 2014, Barcelona (2014), pp.341-347. 
versos resultados negativos como el deterioro de la imagen del país, el debilitamiento de las estructuras gubernamentales para conducir la política exterior, el rezago en temas clave, como el posicionamiento de México en las nuevas corrientes de la economía internacional o los desencuentros con los países vecinos, al norte y al sur, invitaban al distanciamiento del pasado inmediato. ${ }^{3}$

Sin embargo, lejos de trazar ese distanciamiento, el nuevo canciller enfatizó las excelencias de su herencia. "Trabajaremos sobre el legado que hemos recibido... construiremos sobre las amplias y firmes bases de una política exterior seria, profesional, responsable, reconocida y respetada”. Tal reconocimiento, expresado en la Reunión Anual de Embajadores y Cónsules de 2013 (REC 2013) registró bien la prioridad otorgada por el nuevo gobierno a la conciliación con el gobierno anterior. Quedó para otros momentos -que nunca llegaron- una apreciación menos entusiasta pero más objetiva de lo que había recibido como herencia en materia de política exterior el gobierno de EPN.

El mandato que se dio a los embajadores en la REC 2013 estuvo muy lejos de tener como antecedente una planeación de gran calado, requerida para una política exterior que mejorara en el largo plazo el posicionamiento de México en el mundo. Para ello era necesario enfrentar, al menos, las siguientes tareas: a) reordenar las agencias gubernamentales que se ocupan de la política exterior dando responsabilidades precisas a las diversas dependencias y manteniendo una buena coordinación entre ellas, a través de la Secretaría de Relaciones Exteriores; b) establecer una agenda integral de la relación con Estados Unidos acompañada de instancias que den seguimiento, evalúen lo alcanzado y propongan nuevos objetivos cuando parezca necesario; c) remediar el rezago que

${ }^{3}$ Sobre los numerosos temas que generaron polémica o fueron omitidos de la política exterior en época de Calderón, véase Olga Pellicer, "Es momento de mirar hacia afuera”, en Rafael Rodríguez Castañeda (coord.), La agenda pendiente; los desafíos de Enrique Peña Nieto, México, Planeta, 2013, pp. 273-292. 
tenía México en la integración a nuevas corrientes de la economía internacional, en particular las provenientes de Asia, y d) con base en la recién adoptada reforma energética, orientar las relaciones exteriores en materia de petróleo, tomando en cuenta la urgencia de desarrollar conocimientos propios en lo que respecta a la ciencia y tecnología para la exploración y explotación en aguas profundas, la necesidad de diversificar para evitar la dependencia de un solo socio, y el acercamiento a otros países productores que permitiese adquirir experiencias y fortalecer el margen de maniobra de México para moverse en las grandes ligas del mundo del petróleo.

Ninguna de las tareas anteriores se llevó a cabo. El gobierno de Peña Nieto se inclinó por otra manera de actuar. Sin haber delineado un proyecto estratégico para las relaciones de México con el exterior, numerosas delegaciones emprendieron giras al extranjero para promover el potencial de las reformas estructurales, dar a conocer proyectos de envergadura como la construcción de un nuevo aeropuerto para la Ciudad de México o el tren rápido México-Querétaro. En otras palabras, la política exterior tuvo su principal expresión en el llamado entusiasta a los inversionistas para conocer las oportunidades ofrecidas por las reformas estructurales y por los proyectos de inversión auspiciados por el gobierno.

\section{EL FIN DE LAS ILUSIONES}

Pocos días después de que EPN pronunciara un discurso lleno de optimismo en la Asamblea General de la ONU, donde invitó a los países del mundo a seguir el ejemplo de su país "que se atrevió a cambiar", una crisis política en México derrumbó los ánimos triunfalistas. Los acontecimientos de Tlatlaya y Ayotzinapa tuvieron el efecto de sacar a la luz problemas estructurales del sistema político mexicano que sacudieron no solamente a los mexicanos, sino a todos aquellos que desde el exterior se interesaban en nuestro país. 
En realidad, el derrumbe de las ilusiones no fue inesperado. Las circunstancias políticas y económicas que obligaron a hacer un alto en el camino y ver con más realismo los problemas de violencia, corrupción e impunidad que aquejaban al país estaban allí desde hacía tiempo. Pasaron a segundo término en 2013 por la embriaguez que produjo el Pacto por México y la consiguiente posibilidad de aprobar las llamadas reformas estructurales. Pero pronto se advirtió que una cosa era aprobar leyes y reglamentos, y otra muy distinta implementarlas en un ambiente pleno de tensiones políticas que prevalecían en diversas regiones del país. La presencia de grupos de delincuencia organizada que tornaban ingobernables ciertas zonas de Guerrero, Oaxaca y Tamaulipas era conocida. También se conocían los grupos de autodefensa que habían decidido tomar la aplicación de la ley en sus manos ante la insoportable situación creada por el crimen en Michoacán. A nadie sorprendía el caos ahí reinante.

Distintas instituciones internacionales, gubernamentales y no gubernamentales, habían venido señalando desde hacía varios años el contubernio existente entre mandos estatales, alcaldes y policías con el crimen organizado. Habían documentado, también, las desapariciones forzadas cuyo caso más dramático fueron los 72 migrantes asesinados en San Fernando, Tamaulipas. La existencia de fosas clandestinas era un fenómeno conocido.

A lo anterior cabe añadir los datos proporcionados por el INEGI respecto del funcionamiento del sistema de justicia en México y los reveladores estudios del cIDE que confirmaban la desigualdad en la aplicación de la ley y los grados de impunidad que prevalecían a lo largo del país.

A la mayor toma de conciencia sobre tales problemas, propiciada por el auge mediático que adquirió el caso de Ayotzinapa, se sumó el desprestigio del jefe del poder ejecutivo y parte de su gabinete, derivado de investigaciones periodísticas relativas a los tratos sospechosos con inversionistas privilegiados por el gobierno, los cuales indicaban conflictos de intereses y corrupción a gran escala en la elite política. 
Si a todo ello agregamos la pérdida de credibilidad de los partidos políticos -en particular el PRD, por pertenecer a ese partido el presidente municipal presuntamente coludido con el crimen organizado que resultó en la desaparición de los 43 estudiantes de Ayotzinapa-, el problema de la carencia de canales de expresión partidaria del descontento se colocó como un factor que profundizó aún más la crisis política.

Desde el punto de vista de la economía la situación también era problemática. Durante el primer año del gobierno de Peña Nieto, las esperanzas en el "momento mexicano" que permitiría crecer $5 \%$ al año se vieron drásticamente reducidas cuando sólo se alcanzó un modesto crecimiento de 1.2\%. En 2014 las cosas tomaron mejor rumbo, pero con altibajos en el conjunto del sexenio el crecimiento osciló entre $2.1 \%$ y $2.3 \%$. Nunca se alcanzó aquel $5 \%$ prometido. La baja de los precios del petróleo y su lenta recuperación explican, en parte, lo sucedido. ${ }^{4}$

Al finalizar 2014, las preocupaciones más notorias tenían que ver, por una parte, con la posibilidad de superar la crisis política y, por la otra, con el grado en que las reformas estructurales diesen resultados visibles en el corto plazo para dinamizar el crecimiento económico y comenzar a cosechar éxitos útiles para mejorar la imagen de un gobierno que, de nuevo, se encontraba bajo la crítica constante de los medios de comunicación internacionales.

Con mayor insistencia que en el pasado, los acontecimientos de Tlatlaya y Ayotzinapa ocuparon espacios importantes en los medios de comunicación internacionales y en los pronunciamientos de gobiernos, parlamentos y organizaciones internacionales gubernamentales y no gubernamentales. Algunos parlamentarios de la Unión Europea llegaron, incluso, a solicitar que se paralizaran los trabajos para la modernización del Acuerdo de Asociación con México, en tan-

${ }^{4}$ Para un buen recuento de las circunstancias internas que pusieron fin al "momento mexicano, véase Olga Pellicer, "La difícil herencia del 2014” en Proceso, núm. 1991, 28 de diciembre de 2014. 
to no se reconstruyera la confianza en la aplicación efectiva del Estado de derecho y el respeto a los derechos humanos en México.

Fue notable el contraste entre la imagen de México como país soñado para la inversión extranjera y el turismo, que con entusiasmo promovió el gobierno de Peña Nieto, y los problemas de un país donde la desconfianza en las instituciones políticas se estaba generalizando y la violencia se manifestaba bajo sus peores formas. Esa visión contradictoria rompió con el encanto que se quiso construir sobre el atractivo de las reformas estructurales. Visto a la distancia, sorprende que la elite gobernante no advirtiera que, salvo que decidiera dar un golpe de timón contundente para poner fin a la corrupción, reconstruir el Estado de derecho y recuperar la confianza ciudadana en las instituciones del gobierno, se avanzaba hacia un derrumbe del sistema político existente.

Ahora bien, para mediados de 2015, las preocupaciones anteriores pasaron a segundo plano. Una situación por demás conflictiva para México estaba tomando forma en el discurso de uno de los numerosos candidatos presidenciales para las elecciones estadounidenses de 2016. El magnate Donald Trump, una personalidad muy conocida por sus programas televisivos y los edificios y hoteles que llevan su nombre, buscaba la nominación del Partido Republicano para la presidencia de Estados Unidos. Sus discursos contenían ya un elemento xenófobo y antiinmigrante dirigido directamente contra México. Aunque su designación parecía remota, las alarmas comenzaron a sonar en la cancillería mexicana.

\section{TRUMP EN EL HORIZONTE}

A mediados de 2015, ante las noticias relativas al ambiente político-electoral en Estados Unidos, comenzaron a tomarse medidas cuya urgencia no se había considerado. Un primer paso fue remediar omisiones como la de haber esperado seis 
meses para designar a un nuevo embajador en Washington. ${ }^{5}$ La elección recayó en una personalidad ajena al servicio exterior de carrera, buen amigo de EPN, académico distinguido y conocedor de Estados Unidos. Aunque no había trabajado específicamente sobre ello, el embajador Miguel Basáñez tenía interés particular en las comunidades mexicanas establecidas legalmente en Estados Unidos (aproximadamente 30 millones de personas). El punto más destacado de su proyecto como embajador fue buscar el empoderamiento de esas comunidades para que tuviesen voz en las elecciones que se avecinaban. El objetivo era lograr que hiciesen contrapeso a las posiciones antimexicanas promovidas por el candidato republicano, entre otras maneras, mediante acciones de cabildeo en el Congreso.

Los proyectos de Basáñez no tuvieron tiempo de fructificar. Más por motivos internos que por intereses definidos de política exterior, hubo un reemplazo en la Secretaría de Relaciones Exteriores. La nueva canciller fue Claudia Ruiz Massieu, hasta entonces secretaria de Turismo y miembro de uno de los grupos más poderosos del PRI. Ella concedió importancia al fortalecimiento de los consulados en Estados Unidos enviando nuevo personal y ampliando sus funciones para que pudiesen contrarrestar las arengas de Trump en contra del TLCAN y los trabajadores mexicanos. Mantuvo el interés en las comunidades mexicanas y, en general, la relación con políticos y empresarios de dicho país que, por motivos de interés económico, favorecen la relación con México. Siguiendo una línea de coherencia con la diplomacia consular, nombró como embajador a Carlos Sada, quien tenía una larga trayectoria en este servicio en Estados Unidos.

Para entonces, la campaña de Hillary Clinton tenía como uno de sus ejes principales la relación con los grupos hispa-

${ }^{5}$ La lentitud en designar a un embajador sugiere que, desde la perspectiva de los dirigentes políticos, la relación con EEUU la conducen los diversos actores que participan en ella, la acción del gobierno es marginal. Véase Olga Pellicer, "El embajador en Washington", Proceso, núm. 2025, México, 23 de agosto de 2015. 
nos, mayoritariamente mexicanos, que tanto contribuyeron a la segunda victoria electoral de Obama en 2012. Con la prudencia esperada de un embajador frente los asuntos electorales del país en que está adscrito, era claro que el candidato favorito para México era Hillary Clinton. La opinión pública en México resentía ya una profunda antipatía hacia el candidato republicano que profería cotidianamente insultos contra México.

Con tales antecedentes, fue sorpresiva la visita de Trump al país como candidato invitado por el gobierno mexicano. La iniciativa provino del secretario de Hacienda, Luis Videgaray. La amistad personal con el yerno de Trump, Jared Kushner, habría sido el enlace necesario para arreglar dicha visita. Según rumores, la secretaria de Relaciones no estaba enterada.

Serán necesarias investigaciones desde la perspectiva de política burocrática para poder explicar los motivos que llevaron a Videgaray a tomar una decisión de tan alto costo político, la cual fue vivamente criticada por la opinión pública nacional e internacional. Los resultados inmediatos de la visita no modificaron en lo absoluto el discurso fuertemente agresivo contra México que sostenía Trump. El malestar interno en México se hizo sentir en todos los medios de comunicación. A pesar de ser considerado el colaborador más cercano y estimado por EPN, Videgaray se vio obligado a renunciar. ${ }^{6}$

Ante la sorpresa y el malestar de numerosos sectores en todo el mundo, Trump resultó triunfador en las elecciones del 8 de noviembre. En el interior del gobierno de EPN el juego cambió y Luis Videgaray fue nombrado secretario de Relaciones Exteriores. Su labor principal, una de las más complejas que es posible imaginar, era conducir la política

${ }^{6}$ La reprobación nacional de esa invitación se profundizó al conocerse las declaraciones agresivas de Trump contra México, pronunciadas en Arizona pocas horas después de regresar de México. Véase Olga Pellicer, "La mayor amenaza", Proceso, núm. 2079, 4 de septiembre de 2016. 
para mantener un buen entendimiento con el gobierno de Estados Unidos, país con el que, cabe reiterarlo, México se encuentra profundamente vinculado desde el punto de vista de la economía, la seguridad interna y el tejido social. ¿Cómo era posible entenderse con Estados Unidos cuando su nuevo presidente no dejaba de clamar que era necesaria la construcción de un muro para separar a los dos países? ${ }^{7}$

Los meses de enero de 2017 a julio de 2018 tuvieron como el objetivo más importante de la política exterior mantener una imagen de cordialidad hacia Estados Unidos, la cual se buscaría por diversos caminos que pueden agruparse en tres grandes líneas. La primera tiene que ver con los esfuerzos para normalizar el diálogo entre los dos presidentes de manera que se recuperara una relación de respeto y colaboración entre ambos, tarea que estuvo a cargo de Luis Videgaray. La segunda era la negociación en materia comercial. Se partía aquí de una situación muy espinosa, creada por las declaraciones de Trump para quien el TLCAN era "el peor tratado firmado por Estados Unidos”. La tercera fue la relacionada con los temas de migración y seguridad. En ese ámbito, recibió atención especial el tema de los llamados "dreamers", jóvenes que llegaron como niños a Estados Unidos y a quienes Obama había prometido un tratamiento especial para regularizar su situación migratoria.

Los intentos de construir un diálogo cordial entre Trump y EPN, propósito que se convirtió en el leitmotiv de la diplomacia de Videgaray, fueron infructuosos. El primer episodio sirvió de ejemplo. Pocos días después de la toma de posesión de Trump, Videgaray voló a Washington para lograr una visita presidencial de suerte que EPN se convirtiera en el primer mandatario que tenía un encuentro con Trump; la fecha elegida era el 31 de enero. Cierto que hubiese sido significativo lograrlo. Sin duda hubiera contribuido a disipar la enorme

${ }^{7}$ Olga Pellicer, "El primer encuentro”, Proceso, núm. 2101, 5 de febrero de 2017. 
animosidad hacia el vecino del sur que Trump cultivó durante su campaña. ${ }^{8}$

Sin embargo, el intento fue desafortunado. El día de la llegada de los funcionarios mexicanos a Washington, Trump firmó la primera orden ejecutiva para iniciar la construcción del muro y giró instrucciones a la Oficina de Seguridad Nacional para tomar las medidas necesarias para acelerar las deportaciones de migrantes ilegales en Estados Unidos.

Después de una estancia de dos días en que los enviados mexicanos conversaron con miembros de la oficina de la Casa Blanca, Trump publicó varios tuits ofensivos para México, la mayoría relacionados con el pago, por parte de nuestro país, del citado muro. Comprensiblemente, se canceló la visita de EPN a Washington. La “cordialidad” la salvó, hasta cierto punto, una conversación telefónica de casi una hora entre los dos presidentes que abrió el espacio, según un comunicado de la Presidencia de la República, "para que el gobierno de México y el gobierno de Estados Unidos continúen dialogando".

Con variables, los intentos posteriores para preparar una visita de EPN a Washington terminaron con algún incidente desagradable que lo tornó imposible. Los presidentes se encontraron, finalmente, en Buenos Aires a finales de noviembre, dos días antes de que EPN terminase su mandato. Allí se firmó sin mayores ceremonias el texto actualizado del TLCn, denominado ahora Tratado México, Estados Unidos, Canadá (T-MEC por sus siglas en español y USMCA -United States, Mexico and Canada Agreement- por sus siglas en inglés).

$\mathrm{Al}$ parecer, la intensa labor diplomática llevada a cabo por Videgaray no fue del todo infructuosa a los ojos del presidente mexicano. La justificación para otorgar a Kushner la Orden Mexicana del Águila Azteca, el más alto reconocimiento de México a un extranjero, fue el papel que desempeñó ase-

${ }^{8}$ Olga Pellicer, "La diplomacia mexicana en la encrucijada", en Foreign Affairs Latinoamérica, México, IтAм (abril-junio de 2017), pp. 24-31. 
sorando al presidente Trump para que éste no terminara bruscamente con el TLCAN, sino que pusiera en marcha las negociaciones para su actualización.

Está abierto a discusión si fue Kushner, o los intereses de numerosos grupos políticos y económicos en el interior de Estados Unidos que se benefician del comercio con México, lo que pesó más en las decisiones del presidente estadounidense respecto al TLCAN. Lo cierto es que mantener el Tratado era la condición sine qua non para continuar con una buena relación. Las consecuencias de ponerle fin de manera abrupta hubiesen sido extremadamente negativas, provocando una franca desestabilización de la economía mexicana cuyos sectores más dinámicos se encuentran, justamente, en la exportación de manufacturas, en particular de la industria automotriz, hacia Estados Unidos. Igualmente dañino hubiera resultado para numerosas regiones y ciudades de Estados Unidos cuya actividad económica depende, fundamentalmente, del comercio con México.

Las diversas rondas de negociaciones para la modernización del TLGaN fluyeron bien desde el punto de vista del profesionalismo de los grupos técnicos encargados de temas que ya formaban parte del tratado y sólo requerían ajustes para actualizarlos, o bien, de la incorporación de temas nuevos que no tenían un trasfondo político conflictivo, como la nueva modalidad de comercio en línea. La delegación mexicana encabezada por el secretario de Economía, Ildefonso Guajardo, llevó a cabo un trabajo de gran calidad en esos rubros.

Los problemas aparecían cuando la delegación estadounidense ponía sobre la mesa temas abiertamente inaceptables, como la propuesta de someter el tratado a consideración cada cinco años para decidir su permanencia, lo que podía tomarse como una franca provocación, ya que eliminaba el objetivo central de un acuerdo de esa naturaleza que es proporcionar certidumbre a los inversionistas en el largo plazo. Tales propuestas, sumadas a reiteradas declaraciones de Trump sobre la necesidad de revertir el déficit comercial 
que se tiene con México, mantenían vivo el temor de que el tratado fuera bruscamente abandonado por EEUU.

Hacia finales de agosto, es decir a casi dos meses de la elección de Andrés Manuel López Obrador como nuevo presidente en México, la negociación dio un giro inesperado. Se anunció con gran cobertura mediática la firma de un acuerdo entre México y Estados Unidos. ${ }^{9}$ La propaganda distribuida desde la Casa Blanca anunciaba: "América ha volteado la página de un acuerdo comercial que sacrificó nuestra prosperidad, ahuyentó nuestras compañías, nuestros trabajos y el bienestar de nuestra nación”.

A su vez, desde la oficina del representante comercial salieron páginas informativas que señalaban: "El nuevo acuerdo transforma las cadenas productivas para que tengan mayor contenido producido en Estados Unidos, en particular, contenido que es clave para la producción automotriz en el futuro, así como para generar empleos altamente remunerados".

A pesar del ánimo festivo con que se celebró el anuncio, incluido Jesús Seade, el representante de AMLo, se trataba en realidad de un texto preliminar en el que aún era incierta la participación de Canadá y, por consiguiente, la posibilidad de que fuera ratificado en los congresos tanto de México como, sobre todo, de Estados Unidos.

Pasaron algunos meses para que, al fin, habiendo introducido ciertas cláusulas que interesaban particularmente a Canadá, esta nación se sumara al nuevo texto del Tratado. Como ya se señaló, la firma del acuerdo tuvo lugar en Buenos Aires, a finales de noviembre de 2018, aprovechando la presencia allí de los mandatarios de los tres países interesados que asistían a la reunión del G20. Queda abierta la pregunta sobre el comportamiento que tendrá el Parlamento

9 Analistas mostraron escepticismo respecto de las consecuencias a largo plazo de nuevas normas acordadas para el sector automotriz, así como las dificultades que tendrá implementar las relativas a cuestiones laborales. Véase Olga Pellicer, "México-EU; anuncio polémico", Proceso, núm. 2183, 4 de septiembre de 2018. 
canadiense y los Congresos de México y Estados Unidos al momento de considerar la ratificación.

Es complicado definir cuál ha sido la posición mexicana para defender a los trabajadores migrantes, más allá de las actividades de protección que desde hace mucho se vienen llevando a cabo desde los consulados. Recordemos que desde la época de Felipe Calderón se decidió "desmigratizar" la agenda. Es decir, colocar por delante que la política migratoria era un asunto interno de Estados Unidos y a ellos sólo correspondía tomar decisiones al respecto. Se pensó, desde mi punto de vista erróneamente, que ese distanciamiento era útil para no entorpecer las fuerzas internas de Estados Unidos, principal pero no únicamente en el Partido Demócrata, que favorecen una reforma migratoria incluyente. Se creyó, también, que dejar en segundo término conflictos por motivos de migración permitía avanzar en otros frentes, como la agilización de pasos fronterizos o cooperación en el ámbito de la educación.

La llegada al poder de Trump y su marcada animosidad contra los migrantes ha indignado a la sociedad mexicana. No así al gobierno en busca de "diálogo y cordialidad". Situaciones tan escandalosas como la separación de niños de sus padres que siguió a la decisión de convertir en delito la entrada a Estados Unidos sin los documentos requeridos no mereció un reclamo frontal del gobierno de EPN. El problema incluso se minimizó: Videgaray llegó a señalar el hecho de que la mayoría de esos niños no eran mexicanos sino centroamericanos. ${ }^{10}$

La llegada de una ola de caravanas de migrantes centroamericanos que recorren el territorio mexicano en tránsito hacia Estados Unidos, con la intención de solicitar asilo, que seguramente no les será concedido, ha inaugurado un nue-

${ }^{10}$ La voluntad de conciliar con eEuU fue evidente en conferencia de prensa de Videgaray, donde hubo tendencia a minimizar el problema al insistir que la mayoría de los niños no eran mexicanos, sino centroamericanos. Véase Olga Pellicer, "Condenar sin ambivalencias", Proceso, 18 de junio de 2018. 
vo periodo de tensiones y crisis humanitarias en las que se encuentran involucrados, quiéranlo o no, los gobiernos y las sociedades de Estados Unidos, México y los países centroamericanos. Constituye un fenómeno de gran complejidad que se coloca como uno de los grandes problemas a resolver para el gobierno que acaba de tomar posesión en México. En torno a ese tema se han dado los primeros pasos de su administración en materia de política exterior.

\section{LA POLÍTICA EXTERIOR DEL PRESIDENTE ELECTO}

El primero de julio de 2018, los resultados de las elecciones presidenciales dieron un vuelco de proporciones históricas al sistema político mexicano. El partido de Andrés Manuel López Obrador, el Movimiento de Regeneración Nacional (Morena), ganó en elecciones democráticas y transparentes la Presidencia de la República, la mayoría en las dos cámaras del Congreso, la mayoría de las gubernaturas que compitieron, municipios y congresos locales. En su tercer intento por llegar a la presidencia, AMLO se convirtió en la personalidad con mayor poder político que se ha visto en México durante las últimas décadas.

La magnitud del triunfo fue tan contundente, que aceleró los tiempos que por ley transcurren entre ser candidato ganador y presidente. Formalmente, ese cambio ocurriría -como sucedió- el primero de diciembre; en realidad, las circunstancias de 2018 minimizaron la imagen del presidente en funciones, otorgando ya responsabilidades de envergadura al candidato electo. Entre julio y diciembre de 2018, EPN se desdibujó mientras que el entonces todavía presidente electo tomó de hecho las riendas del gobierno mexicano. La política exterior fue el ámbito en que esos hechos se manifestaron con mayor claridad. La media hora de conversación de AMLo con Donald Trump, menos de 48 horas después de conocerse su triunfo electoral, fue una expresión elocuente de la 
aceleración de los tiempos políticos que hicieron de AMLO un presidente en funciones antes de serlo formalmente. ${ }^{11}$

Cabe recordar que la política exterior no fue un tema prioritario durante las campañas electorales. El debate entre los candidatos presidenciales destinado a la política exterior fue de bajísima calidad y evidenció el escaso interés que habían dedicado al tema. No existían, pues, las mejores condiciones para iniciar el diálogo con el presidente del país que, por muchas y distintas razones, es el centro de gravedad para las relaciones exteriores de México.

A pesar de lo anterior, según los reportes de prensa, la conversación telefónica fue positiva en términos del ánimo respetuoso y amigable que mostraron ambas partes. Más allá de un tono cordial, hubo cuestiones de fondo. Por parte de Trump, el señalamiento de que podían ser deseables negociaciones para un acuerdo comercial bilateral México-EeuU. Aunque no se hizo explícito, era claro que el tema de Canadá estaba presente y se trazaba un camino para ejercer presión sobre sus negociadores.

Por parte de AMLO, hubo la propuesta de reducir la migración de mexicanos a Estados Unidos y mejorar la seguridad fronteriza como parte de un proyecto integral de desarrollo que genere empleo en México. La cooperación del gobierno de Trump para ese proyecto sería la aproximación más positiva para el propósito de detener los flujos migratorios. Presentar de inmediato una versión distinta a la de sus antecesores en materia migratoria fue muy congruente con el pensamiento de AMLO, presidente recién electo y un líder de izquierda conocido por su compromiso con las causas sociales.

Pocas semanas después, una delegación de muy alto nivel, encabezada por el canciller de Estados Unidos, Mike Pompeo,

${ }^{11}$ El hecho es que durante la campaña electoral la política exterior no se trabajó con seriedad. No hubo, pues, tiempo para delinear una verdadera estrategia de política exterior. Véase Olga Pellicer, "Los riesgos del apresuramiento", Proceso, núm. 2177, 22 julio de 2018. 
visitó México. Lugar especial en dicha visita tuvo el encuentro del conjunto de la delegación con el presidente electo. De acuerdo con la mayoría de los comentaristas, el gobierno de Trump dejó claro que no es indiferente a lo que ocurre en el país fronterizo que forma parte del perímetro de seguridad de Estados Unidos. Como exdirigente de la cIA, Pompeo conoce muy bien los mecanismos que desde siempre se han utilizado para tener información y ejercer influencia sobre lo que ocurre en México. Desde esa perspectiva, la visita misma a López Obrador fue el mensaje.

En segundo lugar, el encuentro con el presidente electo durante el que se le dio un claro énfasis a las dimensiones de su triunfo electoral fortaleció su legitimidad como interlocutor de política exterior. Abrió aún más la puerta para que se apresuraran los tiempos y que, a sólo dos semanas de su elección, AMLo comenzara a operar como presidente en funciones.

Fue interesante advertir, tanto en la visita de Pompeo a Los Pinos como en el encuentro con el canciller Videgaray, que existía la disposición del gobierno en funciones no sólo de coordinarse con el equipo de quienes tomarían posesión el primero de diciembre, sino de pasarles abiertamente la estafeta.

Durante el encuentro con Pompeo, AmLo entregó un documento dirigido a Trump que, según información proporcionada a la prensa, contenía las bases para mejorar la relación México-Eeuu. Marcelo Ebrard, virtual canciller de López Obrador, informó que las grandes líneas del documento se referían a comercio, desarrollo, migración y seguridad.

La enumeración de esos ejes, sumado a declaraciones hechas durante la campaña y en la conversación telefónica AMLO-Trump permitieron anotar que se trata de introducir en el diálogo con Estados Unidos el principio según el cual no hay solución posible al tema migratorio, ni estabilidad y seguridad en México y Centroamérica, si no hay cooperación para el desarrollo. Importa subrayar la referencia a Centroamérica como elemento estrechamente vinculado 
a los problemas de México; fue un antecedente muy útil para el tratamiento de los hechos que poco después se presentaron.

Algunas semanas después de haberse enviado esa misiva, la llegada de la primera gran caravana de migrantes provenientes de Honduras, formada por aproximadamente 7000 personas, se convirtió en un fenómeno de enormes proporciones con repercusiones muy grandes para la relación con Estados Unidos, para la estabilidad interna de México y para la reflexión y el debate sobre el tema de migración a nivel mundial. ${ }^{12}$

La solución que se encuentre al fenómeno de las caravanas procedentes del llamado Triángulo del Norte (Guatemala, El Salvador y Honduras) que han comenzado a llegar a México de paso a Estados Unidos, con formas de organización novedosas por el número de personas que las integran (más de mil en todos los casos) y la presencia de líderes que los cohesionan y orientan, es uno de los retos más visibles al que deberá encontrar una respuesta el nuevo gobierno de López Obrador.

Cabe recordar que el problema de los migrantes centroamericanos en tránsito por México hacia Estados Unidos no es nuevo en la agenda de situaciones problemáticas entre los dos países. Ocupa un lugar importante en dicha agenda desde el verano de 2014, cuando se elevó bruscamente el número de niños no acompañados que llegaron a Estados Unidos, creando una crisis humanitaria de grandes dimensiones muy comentada en todos los medios de comunicación.

El presidente Obama conversó entonces telefónicamente con EPN solicitando ayuda. Surgió así, bajo los auspicios de la Secretaría de Gobernación, el llamado Proyecto Frontera Sur, cuyo resultado más importante fue la deportación de

12 Tres circunstancias le dan carácter especial a este movimiento migratorio: las dimensiones del grupo, la organización interna y el momento escogido para hacerlo visible. Véase Olga Pellicer, "La Caravana y sus efectos”, Proceso, núm. 2191, 28 de octubre de 2018. 
niños centroamericanos a su país de origen y la aceptación de ayuda, poco visible, de agencias estadounidenses y canadienses en la región. Como señalamos anteriormente, hubo interés en no modificar el discurso según el cual la política migratoria de Estados Unidos era una cuestión interna que sólo correspondía a ellos manejar.

Se sabe, de manera extraoficial, que el gobierno de Trump había insistido con Luis Videgaray para hacer de México un "tercer país seguro", al estilo del acuerdo de Turquía con la Unión Europea. Se trata de ser el país dentro del cual se instalan en albergues, $u$ otras formas de alojamiento, quienes pretenden obtener asilo en Estados Unidos. De no obtenerlo (lo cual es lo más probable dada la política de Trump en la materia), el gobierno mexicano asume la responsabilidad de devolverlos a su país de origen. Trump ofreció a cambio ayuda económica, tanto para los gastos que implica responder a las diversas necesidades de los migrantes mientras están en territorio mexicano, como para el desarrollo en general de la región en donde se concentran.

$\mathrm{Al}$ parecer, semejante propuesta fue discutida por Videgaray con el gobierno electo y descartada. Permanece, sin embargo, como un antecedente que muy probablemente formó parte de las pláticas que dos días después de la toma de posesión de AMLO inició el nuevo secretario de Relaciones Exteriores, Marcelo Ebrard, en Washington. La información sobre tales pláticas apenas se da a conocer al momento de terminar este trabajo.

\section{Conclusiones}

El análisis de la política exterior del gobierno del presidente Enrique Peña Nieto no puede llevarse a cabo independientemente de las vicisitudes de un sexenio en que la evolución de los acontecimientos internos y externos fue modificando las coordenadas dentro de la cuales había -o no- campo de maniobra para la política exterior. 
El camino escogido por EPN para enfrentar los problemas heredados de la administración de Felipe Calderón, entre los que sobresalía la violencia y la ingobernabilidad en algunas regiones del país, fue claramente insuficiente. Cierto que su manejo de prensa para disimular tales problemas fue exitoso, pero sus efectos fueron de muy corta duración: el "momento mexicano" se disipó rápidamente.

La apuesta más importante fue construir alianzas políticas necesarias para llevar a cabo reformas estructurales de carácter constitucional que, sin duda, eran necesarias para romper inercias que entorpecían el desarrollo del país. Sin embargo, los acuerdos cupulares entre partidos y los entendimientos, no carentes de corrupción, con grupos empresariales, así como otros factores de poder permitieron modificar la Constitución, pero no proporcionaron bases firmes para implementar las mencionadas reformas. La conciliación política expresada en el Pacto por México fue abandonada por los sectores de izquierda, que se sintieron traicionados por sus líderes. Nació así el nuevo movimiento social que acabaría por hacer trizas al sistema político existente.

Las reformas estructurales obtuvieron resultados positivos en algunos casos, por ejemplo, en telecomunicaciones. Pero pronto se advirtieron las limitaciones de otras: una reforma energética que debilitaba, en vez de fortalecer, a la empresa mexicana que, dentro de un nuevo esquema de colaboración con inversiones privadas nacionales y extranjeras, seguía siendo indispensable para la seguridad energética del país. Otro caso sobresaliente fue la vulnerabilidad de una reforma educativa que no contaba con un elemento clave para su éxito: la simpatía y el compromiso de los maestros, agrupados en uno de los sindicatos más grandes y poderosos de toda América Latina.

En las condiciones anteriores, sucesos tan dramáticos como los de Tlatlaya o Ayotzinapa, o situaciones tan escandalosas como los actos de corrupción en altos mandos del ejecutivo fueron suficientes para poner fin al optimismo que acompañó los primeros dos años del gobierno de Peña Nie- 
to. Las expresiones específicas de política exterior en esos momentos, durante los cuales se llevaron a cabo múltiples visitas y giras en el exterior, en poco modificaron la curva descendente en la que se precipitaba el gobierno.

A partir de mediados de 2015 un factor externo, como lo fue la aparición del movimiento antimexicano en Estados Unidos, encabezado por Donald Trump, se convirtió en un problema central para el gobierno de México. Sobra insistir en las diversas maneras en que una decisión tomada en el país del norte puede afectar la estabilidad económica y las relaciones políticas y sociales en México. Baste referirnos, por ejemplo, a los efectos sobre los movimientos de la Bolsa o del tipo de cambio peso-dólar que ocurriría si el gobierno estadounidense decide, situación poco probable pero no imposible, cerrar por algunos días los pasos fronterizos.

Es normal, pues, que la sombra de Trump haya dominado las preocupaciones del gobierno mexicano en un periodo que se extiende hasta la actualidad y en el que se entrecruzaron, entre julio y diciembre de 2018, dos presidencias en México, con objetivos y bases de apoyo muy distintas.

La firma del T-MEC, independientemente de los efectos adversos de algunas de sus disposiciones, dio un respiro que permitió iniciar, con menor zozobra desde el punto de vista económico, el sexenio de AMLO. Otros problemas no están resueltos, como los aranceles al acero y al aluminio y los caprichos inesperados de Trump, proclive a tomar medidas económicas perjudiciales para sus socios.

Los temas de migración, lejos de resolverse, han entrado en una etapa más compleja que involucra a los gobiernos y sociedad centroamericanos y coloca en el tablero las propuestas de Trump respecto al papel que desea asignar a México en la solución de problemas en su frontera sur.

Por lo que toca a los acuerdos de seguridad entre los dos países, en parte se relacionan con el tema anterior, pero hay muchos otros puntos que no han sido abordados, sobre los cuales hay una gran secrecía, contraria a cualquier voluntad de transparencia e información a la ciudadanía. Entre esos 
puntos pueden citarse al menos tres: el tráfico de armas para grupos delictivos proveniente de Estados Unidos; la operación de agencias estadounidenses en México para fines de inteligencia, como la DEA, la CIA y el FBI; finalmente, pero no de menor importancia, los acuerdos existentes desde la época de Calderón para la formación de cuadros del Ejército y la Marina mexicanos en Estados Unidos.

Más allá de Estados Unidos, hay varios aspectos de la situación internacional que tienen gran impacto sobre México. Me referiré solamente a dos: el primero incluye los cambios ocurridos en América del Sur que avanza hacia la derecha, dejando en soledad al único gobierno de izquierda entre los países grandes de la región. El segundo, la disputa comercial entre Estados Unidos y China, cuya evolución y resultados afectarán las posibilidades de que México diversifique, aunque sea en forma reducida, su gran vulnerabilidad económica frente a Estados Unidos.

Con las reflexiones anteriores en mente, ¿cuál es el dilema más grande que se percibe para la política exterior de AMLO? El dilema de la política exterior al iniciarse la deseada “cuarta transformación” es cómo conciliar esa aspiración con la dificultad de reducir la vulnerabilidad tan diversa y profunda que tenemos frente a Estados Unidos.

\section{Bibliografía}

"El posicionamiento internacional de México en el segundo decenio del siglo Xxı", en Ricardo Lagos y Enrique Iglesias (coords.), América Latina, China y Estados Unidos. Perspectivas latinoamericanas, Chile, Fondo de Cultura Económica, 2015, pp. 213-272.

"La política exterior de México bajo un nuevo presidente", Centro de Estudios Internacionales de Barcelona, сіDов, Anuario Internacional 2014, Barcelona, 2014, pp. 341-347.

Pellicer, Olga, “Condenar sin ambivalencias”, Proceso, 18 de junio de 2018. 
Pellicer, Olga, "El embajador en Washington", Proceso, núm. 2025, 23 de julio de 2015.

Pellicer, Olga, "El primer encuentro", Proceso, núm. 2101, 5 de febrero de 2017.

Pellicer, Olga, "Es momento de mirar hacia afuera", en Rafael Rodríguez Castañeda (coord.), La agenda pendiente; los desafíos de Enrique Peña Nieto, Planeta, 2013, pp. 273-292.

Pellicer, Olga, "La Caravana y sus efectos” Proceso, núm. 2191, 28 de octubre de 2018.

Pellicer, Olga, "La difícil herencia del 2014” en Proceso, núm. 1991, 28 de diciembre de 2014.

Pellicer, Olga, "La diplomacia mexicana en la encrucijada", en Foreign Affairs Latinoamérica, IтAм (abril-junio de 2017), pp. 24-31.

Pellicer, Olga, "La mayor amenaza”, Proceso, núm. 2079, 4 de septiembre de 2016.

Pellicer, Olga, "Los riesgos del apresuramiento", Proceso, núm. 2177, 22 de julio de 2018.

Pellicer, Olga, "México-Eu; anuncio polémico", Proceso, núm. 2183, 4 de septiembre de 2018. 
